\title{
Martensitic transformation under impact with high strain rate
}

\author{
Song Shun-cheng ${ }^{\mathrm{a}, *}$, Ding Gui-bao ${ }^{\mathrm{a}}$, Duan Zu-ping ${ }^{\mathrm{b}}$ \\ ${ }^{a}$ Southwest JiaoTong University, Chengdu 61003, People's Republic of China \\ ${ }^{\mathrm{b}}$ Institute of Mechanics, CAS, Beijing 10080, People's Republic of China
}

Received 23 June 2000; received in revised form 17 March 2001

\begin{abstract}
This paper deals with the quantitative prediction of the volume fraction of martensitic transformation in a austenitic steel that undergoes impact with high strain rate. The coupling relations between strain, stress, strain rate, transformation rate and transformed fraction were derived from the OTC model and modified Bodner-Partom equations, where the impact process was considered as an adiabatic and no entropyincreased process (pressure $\leqslant 20 \mathrm{GPa}$ ). The one-dimensional results were found to model and predict various experimental results obtained on 304 stainless steel under impact with high strain rate. (C) 2001 Published by Elsevier Science Ltd.
\end{abstract}

\section{Introduction}

During the last two decades, it has been unequivocally established that stress or deformationinduced martensitic transformation can significantly enhance the tensile ductility and the fracture toughness of materials.

The common knowledge about the phenomenon of the deformation-induced martensitic transformations is that the stable nucleation sites are initially promoted at the intersection of localized dislocation bands or twins, and then these nuclei evolve into larger volumetric martensites under the loading. Therefore, the constitutive equations of the material must be given to describe the stress or strain-induced martensitic transformations. On the contrary, the martensitic evolving process must affect to the relation between stress and strain.

A constitutive model which takes into account the deformation-induced martensitic transformation in metastable steels has recently been proposed by Stringfellow et al. [1] and the model was developed by Diani et al. [2] and Grujicic et al. [3], but these works which are based on the static Von Mises yield criterion could not illustrate the effects of the deformation rate in

\footnotetext{
*Corresponding author.
} 
impact. In this paper we dealt with the quantitative prediction of the volume fraction of martensitic transformation in a austenitic steel that undergoes a impact loading. The coupling relations between strain, stress, strain rate, transformation rate and transformed fraction were derived from the OTC model and modified Bodner-Partom (B-P) equations, where the impact process $\left(\dot{\varepsilon} \geqslant 10^{2} / \mathrm{s}\right)$ was considered as an adiabatic and no entropy-increased process (pressure $\leqslant 20 \mathrm{GPa}$ ) because under this condition the adiabatic curve is very closed to the isoentropic curve [4]. Finally, in this paper the one-dimensional results were found to model and predict various experimental results obtained on 304 stainless steel under impact with high strain rate.

\section{Statistic of martensitic nucleation and evolution}

A decade ago, Olson et al. [5] introduced a statistical model (OTC model) based on phenomenology and experimental data of Cech and Turnbull to describe the distribution of nucleation site potencies in $\mathrm{Fe}-30 \mathrm{Ni}$. According to this model the transformed martensitic fraction $f$ in material is equal to the probability $p$ in the following way:

$$
f=p=1-\exp \left(-N_{v} V_{p}\right)
$$

where $V_{p}$ is a volumetric number and $N_{v}$ represents the number density of the nucleation sites randomly distributed throughout the material. From the available transformation thermodynamics data, it was determined that the number density $N_{v}$ was related to the Gibbs free energy change, $\Delta g$, which accompanies the martensitic transformation. Next, the martensitic evolving also relied on the elastic interaction of the nucleus with superdislocation-like linear defects, i.e.:

$$
N_{v}=N_{v}^{0} \exp \left[-\alpha \frac{2 \Gamma / \rho}{\Delta g-g^{\mathrm{el}}-w_{\mathrm{f}}}\right],
$$

where $\alpha$ is a constant distribution shape factor, and $N_{v}^{0}$ is the total number density of defects of all potencies, $\Gamma$ is the nucleus/matrix specific interfacial energy, $\rho$ is the planar atomic density of the close packed planes, $g^{\text {el }}$ is the elastic nucleus misfit strain energy, and $w_{\mathrm{f}}$ the frictional work of nucleus/matrix interfacial motion. The total Gibbs free energy change is the sum of the chemical contribution $\Delta g^{\text {ch }}$ and the mechanical contribution $\Delta g^{\sigma}$.

If the changes of $2 \Gamma / \rho, \Delta g^{\mathrm{ch}}, g^{\mathrm{el}}$ and $w_{\mathrm{f}}$ remain small in the impact process, their values can be represented by their mean values and from Eq. (2.2) it is yielded that:

$$
\dot{N}_{v}=\frac{\Delta \dot{g}^{\sigma} N_{v}^{0} \phi_{1}}{\left(\phi_{2}+\Delta g^{\sigma}\right)^{2}} \exp \left(-\frac{\phi_{1}}{\phi_{2}+\Delta g^{\sigma}}\right)
$$

where $\phi_{1}=\langle 2 \alpha \Gamma / \rho\rangle, \phi_{2}=\left\langle\Delta g^{\mathrm{ch}}-g^{\mathrm{el}}-w_{\mathrm{f}}\right\rangle$. Therefore, the transformation rate of martensitic fraction in the impact process can be expressed by

$$
\dot{f}=\frac{\Delta \dot{g}^{\sigma} N_{v}^{0} \phi_{1} V_{p}}{\left(\phi_{2}+\Delta g^{\sigma}\right)^{2}} \exp \left(-\frac{\phi_{1}}{\phi_{2}+\Delta g^{\sigma}}\right) \exp \left(-V_{p} N_{v}\right) \text {. }
$$


This equation illustrates the relations between martensitic fraction change per unit time $\dot{f}$ and mechanical driving force, $\Delta g^{\sigma}$ and its rate, $\Delta \dot{g}^{\sigma}$. From this equation it is shown that $\dot{f}$ relies on the product of two Boltzmann factors, which is consistent with the Becker recrystallized theory [6].

Under the assumption that the process of impact-induced martensitic transformation is Markov process, the transition probabilities for initial and $(n+1)$ th step are expressed respectively by

$$
\begin{aligned}
& p_{0}=1-\exp \left[-V_{p} N_{v}(0)\right], \\
& p_{n}=p_{n-1}+\int_{t}^{t+\Delta t} \dot{f} \mathrm{~d} t
\end{aligned}
$$

and the martensitic fraction for $(n+1)$ th step can be given by

$$
\begin{aligned}
& f_{n+1}=f_{n}+\Delta f_{n}, \\
& \Delta f_{n}=\left(1-f_{n}\right) p_{n} .
\end{aligned}
$$

\section{Impact mechanical driving force and plastic strain}

As mentioned above, the mechanical driving force, $\Delta g^{\sigma}$, is the only variable versus time in impact-induced transformation process. The $\Delta g^{\sigma}$ is a nucleus orientation dependent quantity and can be expressed in terms of the stress normal to the transformation habit plane, $\sigma_{n}$, and the shear stress in this plane, $\tau$, i.e.:

$$
\Delta g^{\sigma}=\sigma_{n} \varepsilon_{0}+\tau \gamma_{0}
$$

where $\varepsilon_{0}$ and $\gamma_{0}$ are the normal and shear transformation strain, respectively. If the stress tensor in global coordinate system is denoted by $\mathbf{T}$, the normal and shear stresses can be written by

$$
\begin{aligned}
& \sigma_{n}=\mathbf{N}_{\text {glob }}: \mathbf{T}, \\
& \tau=\mathbf{R}_{\text {glob }}: \mathbf{T},
\end{aligned}
$$

where $\mathbf{N}_{\text {glob }}$ and $\mathbf{R}_{\text {glob }}$ are transformation tensor in global coordinate system and defined in terms of the Euler angles $(\theta, \varphi, \psi)$ :

$$
\begin{aligned}
& \mathbf{N}_{\text {glob }}(\theta, \varphi, \psi)=\mathbf{P}(\theta, \varphi, \psi) \mathbf{N}_{\text {local }} \mathbf{P}^{\mathrm{T}}(\theta, \varphi, \psi), \\
& \mathbf{R}_{\text {glob }}(\theta, \varphi, \psi)=\mathbf{P}(\theta, \varphi, \psi) \mathbf{R}_{\text {local }} \mathbf{P}^{\mathrm{T}}(\theta, \varphi, \psi),
\end{aligned}
$$


where $\mathbf{R}_{\text {local }}$, the symmetric part of the Schmid tensor, and $\mathbf{N}_{\text {local }}$ are two tensors defined by the habit plane normal $\mathbf{n}$ and shear direction $\mathbf{b}$ in local coordinate system:

$$
\begin{aligned}
& \mathbf{N}_{\text {local }}=\mathbf{n} \otimes \mathbf{n}, \\
& \mathbf{R}_{\text {local }}=\frac{1}{2}[(\mathbf{n} \otimes \mathbf{b})+(\mathbf{b} \otimes \mathbf{n})] .
\end{aligned}
$$

The local to global transformation matrix $\mathbf{P}(\theta, \varphi, \psi)$ is expressed by

$$
\mathbf{P}=\left[\begin{array}{ccc}
c_{1} c_{2} c_{3}-s_{1} s_{2} & -c_{3} s_{2}-c_{1} s_{3} c_{2} & s_{1} c_{2} \\
c_{1} c_{3} s_{2}+s_{3} c_{2} & -s_{2} c_{1} s_{3}+c_{2} c_{3} & s_{1} s_{2} \\
-c_{3} s_{1} & s_{1} s_{3} & c_{1}
\end{array}\right],
$$

where $c_{1}=\cos \theta, c_{2}=\cos \phi, c_{3}=\cos \psi, s_{1}=\sin \theta, s_{2}=\sin \phi, s_{3}=\sin \psi$.

In the same way, the plastic strain caused by the martensitic transformation of a nucleus with some orientation can be written by:

$$
\boldsymbol{\varepsilon}^{\mathrm{p}}(\theta, \phi, \psi)=\gamma_{0} \mathbf{R}_{\mathrm{glob}}+\varepsilon_{0} \mathbf{N}_{\mathrm{glob}}
$$

Under the assumption of a random distribution of nucleation site, the increase in the macroscopic plastic strain, $\Delta \boldsymbol{\varepsilon}_{n}^{\mathrm{p}}$ from $n$th to $(n+1)$ th step in the impact process can be obtained by summing the contribution of all transformed nucleus as follow:

$$
\Delta \varepsilon_{n}^{\mathrm{p}}=\Delta f_{n} \frac{\int_{0}^{2 \pi} \int_{0}^{2 \pi} \int_{0}^{\pi} \varepsilon_{i, n+1}^{\mathrm{p}}(\theta, \phi, \psi) p_{i, n+1}(\theta, \phi, \psi) \sin \theta \mathrm{d} \theta \mathrm{d} \phi \mathrm{d} \psi}{\int_{0}^{2 \pi} \int_{0}^{2 \pi} \int_{0}^{\pi} p_{i, n+1}(\theta, \phi, \psi) \sin \theta \mathrm{d} \theta \mathrm{d} \phi \mathrm{d} \psi}
$$

where $\Delta f_{n}$ can be obtained from Eq. (2.6b), $\varepsilon_{i, n+1}^{\mathrm{p}}$ is the plastic strain of the $i$ th transformed nucleation site at $(n+1)$ th step in impact process and is defined by Euler angles $(\theta, \phi, \psi), p_{i, n+1}$ is the probability that the $i$ th nucleation site operates transformation at $(n+1)$ th step and it is the loading history dependent as

$$
\begin{aligned}
& p_{i, n+1}(\theta, \phi, \psi)=\bar{p}_{n} \exp \left(-\frac{2 \alpha \Gamma / \rho}{\Delta g^{\mathrm{ch}}+\Delta g_{n+1}^{\sigma}(\theta, \phi, \psi)-g^{\mathrm{el}}-w_{\mathrm{f}}}\right), \\
& \bar{p}_{n}=\prod_{i=0}^{n}\left[1-\exp \left(-\frac{2 \alpha \Gamma / \rho}{\Delta g^{\mathrm{ch}}+\Delta g_{n+1}^{\sigma}(\theta, \phi, \psi)-g^{\mathrm{el}}-w_{\mathrm{f}}}\right)\right] .
\end{aligned}
$$

With the above relations the macroscopic plastic strain caused by transformed fraction at $(n+1)$ th step in impact process can be obtained as

$$
\varepsilon_{n+1}^{\mathrm{p}}=\varepsilon_{n}^{\mathrm{p}}+\Delta \varepsilon_{n}^{\mathrm{p}}
$$

and the initial condition read:

$$
\boldsymbol{\varepsilon}_{0}^{\mathrm{p}}=f_{0} \frac{\varepsilon_{0}}{3} \mathbf{I}
$$


where $f_{0}$ is the initial fraction of martensitic nucleus, $\varepsilon_{0}$ is the dilatational plastic strain caused by the complete martensitic transformation (i.e. $f=1$ ).

Furthermore the plastic strain tensor, $\boldsymbol{\varepsilon}_{n+1}^{\mathrm{p}}$, in Eq. (3.9) can be decomposed into its deviatoric and hydrostatic parts as

$$
\boldsymbol{\varepsilon}_{n+1}^{\mathrm{p}}=\sqrt{\frac{3}{2}} \bar{\varepsilon}_{n+1}^{\mathrm{p}} \mathbf{N}+\varepsilon_{h, n+1} \mathbf{I},
$$

where $\bar{\varepsilon}_{n+1}^{\mathrm{p}}$ is the equivalent plastic strain. If $\mathbf{T}^{\prime}$ represents the deviatoric part of the Kirchhoff stress, the flow direction tensor $\mathbf{N}=\mathbf{T}^{\prime} /\left\|\mathbf{T}^{\prime}\right\|$. In Eq. (3.11), $\varepsilon_{h, n+1}$ is the dilatation plastic strain and it reads:

$$
\varepsilon_{h}=\frac{\varepsilon_{0}}{3} f .
$$

\section{Constitutive equations for impact-induced transformation}

In order to describe the stress strain relationship in impact process, the equation in the rate form is written by

$$
\stackrel{\nabla}{\mathbf{T}}=\mathbf{L}^{\mathrm{e}}\left(\mathbf{D}-\mathbf{D}^{\mathrm{p}}\right),
$$

where $\mathbf{T}$ represents Kirchhoff stress tensor and $\stackrel{\nabla}{\mathbf{T}}$ is its Jaumann derivate. $\mathbf{D}$ and $\mathbf{D}^{\mathrm{p}}$ represent the total strain rate tensor and plastic part, respectively. $\mathbf{L}^{\mathrm{e}}$ is the fourth order elastic tensor written by

$$
\mathbf{L}^{\mathrm{e}}=2 G\left(\mathbf{J}-\frac{1}{3} \mathbf{I} \otimes \mathbf{I}\right)+B(\mathbf{I} \otimes \mathbf{I}),
$$

where $\mathbf{J}$ is the fourth order identity tensor, $\mathbf{I}$ is the second order identity tensor, $G$ and $B$ are elastic shear modulus and bulk modulus, respectively and they are defined using a weighted average of the corresponding quantities for two constituent phases. In general, $G$ and $B$ are functions of transient temperature but in this paper they are considered as functions of plastic work under the above assumption that the process of impact is adiabatic and no entropy increased process.

Using the Voigt upper bound model, the plastic strain rate for transforming phase ( $\beta$ phase) is equal to matrix phase ( $\gamma$ phase) i.e. $\mathbf{D}_{\beta}^{\mathrm{p}}=\mathbf{D}_{\gamma}^{\mathrm{p}}=\mathbf{D}^{\mathrm{p}}$ and the B-P equations are introduced as

$$
\frac{1}{2} \mathbf{D}^{\mathrm{p}}: \mathbf{D}^{\mathrm{p}}=D_{0}^{2} \exp \left[-\left(\frac{Z^{2}}{J_{2}^{2}}\right)^{n}\right]
$$

where $D_{0}$ is the limit of the plastic strain rate and $J_{2}$ is the second invariable of stress deviatoric tensor and $Z$ is expressed by

$$
Z=Z_{1}+\left(Z_{0}-Z_{1}\right) \exp \left(-m_{0} w^{\mathrm{p}} / Z_{0}\right),
$$

where $Z_{1}, Z_{0}$ and $m_{0}$ are material parameters. $w^{\mathrm{p}}$ is specific plastic work expressed by

$$
w^{\mathrm{p}}=\Delta g^{\sigma}, \dot{w}^{\mathrm{p}}=\mathbf{T}: \mathbf{D}^{\mathrm{p}} .
$$

In Eq. (4.3), Bodner and Partom took $n$ as that $n=\left(\theta_{\text {melt }} / \theta\right)+b$, where $b$ is a material parameter, $\theta$ is the transient temperature, and $\theta_{\text {melt }}$ is the melt temperature of material. As mentioned above, the process of impact is adiabatic and no entropy increased process, so $n$ can be expressed as a 
function of plastic work and reads:

$$
n=\frac{n_{\mathrm{a}} E_{\mathrm{a}}}{\Delta g^{\sigma}+E_{\mathrm{a}}}+b
$$

where $E_{\mathrm{a}}$ is the material initial specific internal energy at ambient temperature, $\theta_{\mathrm{a}}$, and $n_{\mathrm{a}}=\theta_{\text {melt }} / \theta_{\mathrm{a}}$.

From Eq. (4.3) and the plastic theory the plastic strain rate tensor is written by

$$
\mathbf{D}^{\mathrm{p}}=\left[\left(\frac{1}{2} \mathbf{D}^{\mathrm{p}}: \mathbf{D}^{\mathrm{p}}\right) / J_{2}\right]^{1 / 2} \mathbf{T}^{\prime}
$$

In transforming phase the $\mathbf{D}_{\beta}^{\mathrm{p}}$ can be decomposed as

$$
\mathbf{D}_{\beta}^{\mathrm{p}}=\sqrt{\frac{3}{2} \dot{\bar{\varepsilon}}} \overline{\mathrm{p}}_{\beta} \mathbf{N}+\dot{\varepsilon}_{\mathrm{h} \beta} \mathbf{I},
$$

where $\mathbf{N}=\mathbf{T}^{\prime} /\left\|\mathbf{T}^{\prime}\right\|$, I is the second order identity tensor, and $\dot{\bar{\varepsilon}}_{\beta}$ and $\dot{\varepsilon}_{\mathrm{h} \beta}$ are the equivalent plastic strain rate and dilatational plastic strain rate, respectively in the transforming phase. From the Eq. (4.8), the increment of the plastic strain in transforming phase reads:

$$
\Delta \varepsilon_{\beta}^{\mathrm{p}}=\sqrt{\frac{3}{2}} \bar{\varepsilon}_{\beta}^{\mathrm{p}} \Delta t \mathbf{N}+\dot{\varepsilon}_{\mathrm{h} \beta} \Delta t \mathbf{I} .
$$

In matrix phase ( $\gamma$ phase) since the plastic deformation is caused by slip or twinning, there is no accompanying volume change and $\mathbf{D}_{\gamma}^{\mathrm{p}}$ is written as

$$
\mathbf{D}_{\gamma}^{\mathrm{p}}=\sqrt{\frac{3}{2} \dot{\mathrm{\varepsilon}}} \mathrm{p} \mathbf{N}
$$

i.e.

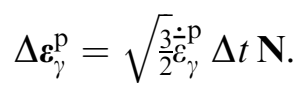

Taking a weighted average of the expressions given by Eqs. (4.9) and (4.10b) and in terms of Eq. (3.12) the plastic strain tensor in two phase material can be written as

$$
\Delta \boldsymbol{\varepsilon}^{\mathrm{p}}=\mathbf{D}^{\mathrm{p}} \Delta t=\sqrt{\frac{3}{2}} \Delta \bar{\varepsilon}^{\mathrm{p}} \mathbf{N}+f \frac{\varepsilon_{0}}{3} \Delta f \mathbf{I}
$$

If the Kirchhoff stress tensor is $\mathbf{T}_{0}$ at time $t$ in impact process, the updated stress tensor $\mathbf{T}$ at new time $t+\Delta t$ is given by

$$
\begin{aligned}
& \mathbf{T}=\mathbf{T}_{0}+\Delta \mathbf{T}, \\
& \Delta \mathbf{T}=\int_{t}^{t+\Delta t} \stackrel{\nabla}{\mathbf{T}} \mathrm{d} t .
\end{aligned}
$$

The Eq. (4.12b) can be evaluated numerically using the generalized trapezoidal rule as

$$
\Delta \mathbf{T}=\eta \stackrel{\nabla}{\mathbf{T}} \Delta t+(1-\eta) \stackrel{\nabla}{\mathbf{T}}_{0}
$$

Using Eq. (4.1) and Euler backward difference method in which $\eta=1$ the stress tensor $\mathbf{T}$ is expressed by

$$
\mathbf{T}=\mathbf{T}_{0}+\Delta t \mathbf{L}^{\mathrm{e}} \mathbf{D}-\Delta t \mathbf{L}^{\mathrm{e}} \mathbf{D}^{\mathrm{p}}
$$


i.e.

$$
\mathbf{T}=\mathbf{T}_{0}+\mathbf{L}^{\mathrm{e}} \Delta \boldsymbol{\varepsilon}-\mathbf{L}^{\mathrm{e}} \Delta \boldsymbol{\varepsilon}^{\mathrm{p}} .
$$

Substituting Eqs. (4.11) and (4.2) into the above equation and considering $\mathbf{N}: \mathbf{I}=0$, the stress tensor $\mathbf{T}$ is recast as

$$
\mathbf{T}=\mathbf{T}_{0}+\mathbf{L}^{\mathrm{e}} \Delta \boldsymbol{\varepsilon}-\sqrt{6} G \dot{\bar{\varepsilon}} \Delta t \mathbf{N}-B f \varepsilon_{0} \dot{f} \Delta t \mathbf{I} .
$$

It is shown from the above derivations that the material constitutive equations for impact-induced transformation are coupling equations of several variables such as $f, \dot{f}, \mathbf{D}^{\mathrm{p}}$ and $\mathbf{T}$, etc., therefore the numerical method must be used to obtain the fraction of martensitic transformation or the strain stress relationship in an impact process.

\section{One-dimensional calculations and comparison with experiments}

In one-dimensional calculations, supposing the orientation of martensitic nuclei transforming to be consistent with the global coordinates i.e. $(\theta, \phi, \psi)=(0,0,0)$, the governing equations are written by

$$
\begin{aligned}
& v_{x 1}=v_{0}, \quad v_{x 2}=0, \\
& \dot{\varepsilon}_{x}=\frac{\partial v_{x}}{\partial x}=\varepsilon_{x}^{\mathrm{e}}+\varepsilon_{x}^{\mathrm{p}}, \\
& \dot{\varepsilon}_{x}^{\mathrm{p}}=\frac{2 D_{0} \sigma_{x}}{\sqrt{3}\left|\sigma_{x}\right|} \exp \left[-\frac{1}{2}\left(3 Z^{2} / \sigma_{x}^{2}\right)^{n}\right] \\
& n=\frac{n_{\mathrm{a}} E_{\mathrm{a}}}{\Delta g^{\sigma}+E_{\mathrm{a}}}+b, \quad b=0, \quad \Delta \dot{g}^{\sigma}=\sigma_{x} \dot{\varepsilon}_{x}^{\mathrm{p}}, \\
& Z=Z_{1}+\left(Z_{0}-Z_{1}\right) \exp \left(m_{0} \Delta g^{\sigma}\right), \\
& \dot{f}=\frac{\Delta \dot{g}^{\sigma} N_{v}^{0} \phi_{1} V_{p}}{\left(\phi_{2}+\Delta g^{\sigma}\right)^{2}} \exp \left(-\frac{\phi_{1}}{\phi_{2}+\Delta g^{\sigma}}\right) \exp \left(-V_{p} N_{v}\right), \\
& f_{0}=p_{0}=1-\exp \left[-V_{p} N_{v}(0)\right] \text {, } \\
& N_{v}=N_{v}^{0} \exp \left(\frac{-\phi_{1}}{\phi_{2}+\Delta g^{\sigma}}\right), \quad N_{v}(0)=N_{v}^{0} \exp \left(\frac{-\phi_{1}}{\phi_{2}}\right), \\
& p_{k}=p_{k-1}+\int_{t}^{t+\Delta t} \dot{f} \mathrm{~d} t, \\
& f_{k+1}=f_{k}+\Delta f_{k}, \quad \Delta f_{k}=\left(1-f_{k}\right) p_{k}, \\
& \Delta \sigma_{x}=2(1+\mu) G \dot{\varepsilon}_{x} \Delta t-G \frac{2 \sqrt{6} \sigma_{x}\left|\dot{\varepsilon}_{x}^{\mathrm{p}}\right| \Delta t}{3\left|\sigma_{x}\right|}-\frac{2(1+\mu)}{(1-2 \mu)} G f \varepsilon_{0} \dot{f} \Delta t,
\end{aligned}
$$






Fig. 1. The relation between the martensite fraction and final impact plastic strain.

where $\mu$ is Poisson's ratio, $v_{x}$ is displacement velocity, and $v_{x 1}$ and $v_{x 2}$ represent displacement velocity conditions at two ends of specimen. The other notations are same as above statements.

It is noted that if the stress $\sigma_{x}$ in Eqs. (5.3)-(5.5) is normalized by static yield stress, the parameters $Z_{1}, Z_{0}$ and $m_{0}$ become dimensionless. In this paper, for analysis of the impact deformed behavior and martensitic transformations of 304-type stainless steel under impact with high strain rate, the parameters $Z_{1}, Z_{0}$ and $m_{0}$ are given as that $Z_{1}=2.07, Z_{0}=0.53, m_{0}=3.45$. According to Refs. [3,5], the total number density of defects $N_{v}^{0}=5.0 \times 10^{12} / \mathrm{m}^{3}$ and the volumetric $V_{p}$, the mol density $\rho_{\mathrm{m}}$ are given as that $V_{p}=9.8 \times 10^{-16} \mathrm{~m}^{3}$, and $\rho_{\mathrm{m}}=3.2 \times 10^{6} \mathrm{~mol} / \mathrm{m}^{3}$.

Though $\phi_{1}$ and $\phi_{2}$ have clearly physical essences, there is some difficulty for us to measure them. Therefore, these parameters are determined by cycling numerical calculations in the light of the experimental results of Staudhammer et al. [7]. The range of the parameters value is first determined. It can be seen from Eq. (5.6) that the order of $\phi_{2}$ should be about same as the order of the plastic work $\Delta g^{\sigma}$. We can see from impact experiments that the order of plastic work is $10^{8} \mathrm{~J} / \mathrm{m}^{3}$ at strain rate $10^{3} / \mathrm{s}$. Considering the high strain rate $10^{3}-10^{4} / \mathrm{s}$, the order of $\phi_{2}$ should be $10^{8}-10^{9} \mathrm{~J} / \mathrm{m}^{3}$ i.e. $10^{2}-10^{3} \mathrm{~J} / \mathrm{mol}$. According to the fact that the martensite fraction is equal to zero for $\Delta g^{\sigma}=0, \phi_{1}$ is larger than $\phi_{2}$.

After the value of parameters is chosen in the range of the parameter value, we can calculate the martensite fraction for the given strain rate and impact time through Eqs. (5.1)-(5.11). So with different strain rate but same impact time we can obtain a curve of martensite fraction versus impact strain. In general the first calculations could not bear comparison with the experimental results, $\phi_{1}$ and $\phi_{2}$ need to be adjusted until the calculated curve is close to the experimental curve. Clearly, this is different to the simple data fitting though the technique is straightforward, because the same parameters must fit various strain rates in the range $10^{3}-10^{4} / \mathrm{s}$.

Through cycling calculations, it is determined that $\varphi_{1}=5830 \mathrm{~J} / \mathrm{mol}$ and $\varphi_{2}=925 \mathrm{~J} / \mathrm{mol}$ in this paper, and in so doing the computational results of the martensitic fraction versus the final impact plastic strain could bear comparison with the experimental results as shown in Fig. 1. The agreement between computational results and experimental results illustrates that the present 


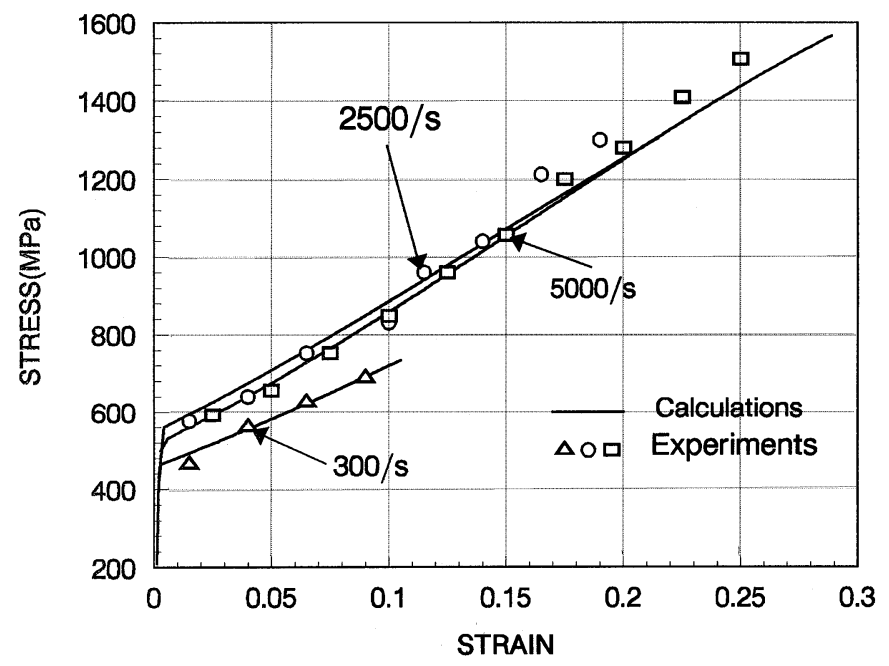

Fig. 2. Strain-stress relationship for 304 steel at various strain rates.

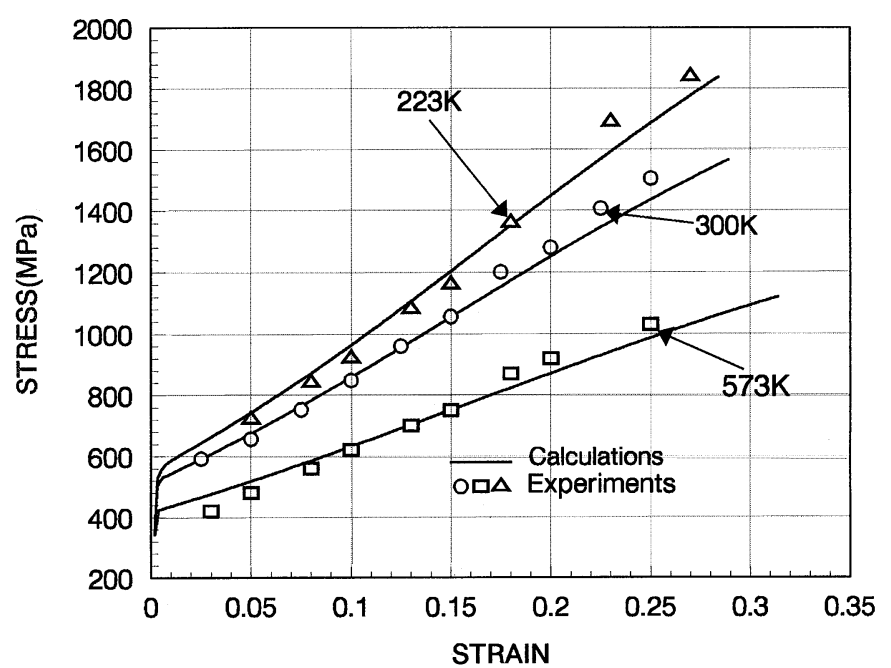

Fig. 3. Strain-stress relationship for 304 steel at various temperatures.

formulations could account for the basic statistics and thermodynamic aspects of impact-induced martensites.

The deformed behavior experiments of 304-type stainless steel are performed with Hopkinson bar instrument that has found a wide acceptance for strain rate testing $\left(10^{2}-10^{4} / \mathrm{s}\right)$. The results of the computations with above parameters and the experiments for strain-stress relationship at various strain rates and various ambient temperatures are shown in Figs. 2 and 3. It can be seen from Fig. 2 that in the range of lower strain rate $(\dot{\varepsilon} \leqslant 2500 / \mathrm{s})$ there is an obvious effect of strain rate on dynamic yield stress, i.e. the yield stress rises with the increase of strain rate, but in the 


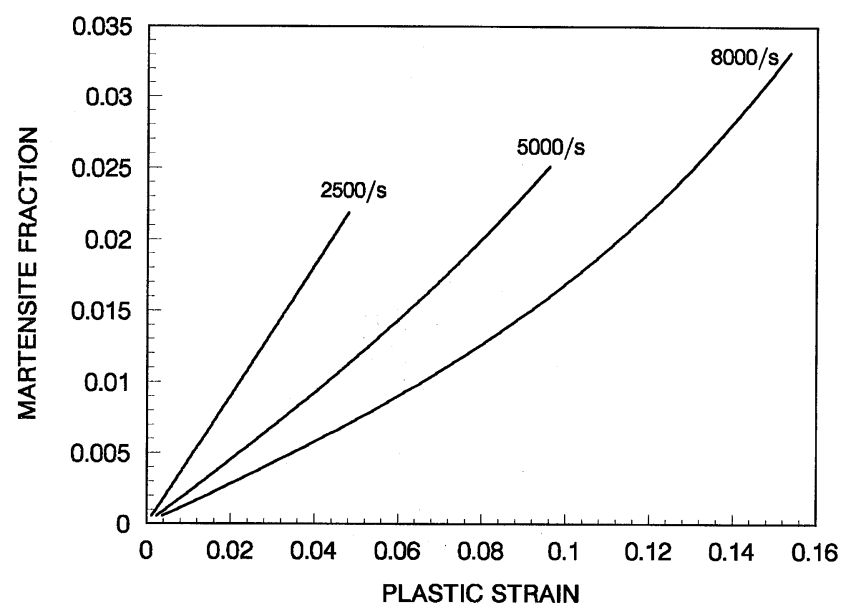

Fig. 4. The evolution of martensite fraction at various strain rates.

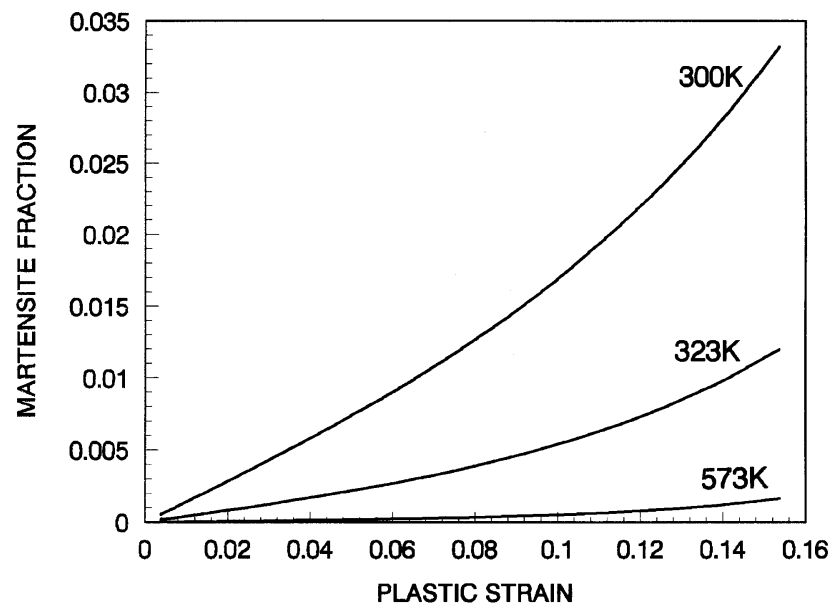

Fig. 5. The evolution of martensite fraction at various temperatures.

range of higher strain rate this is not always the case such as the yield stress at strain rate $5000 / \mathrm{s}$ is slightly less than at strain rate 2500/s. It can be seen from Fig. 3 that the yield stress falls off obviously with the rise in temperature.

The evolution of martensite in 304-type steel as a function of the plastic strain at various impact strain rates and at the same strain rate $(\dot{\varepsilon}=8000 /$ s $)$ but various temperatures is computed with presented formulations and parameters and the results are depicted in Figs. 4 and 5, respectively. As expected it is shown in Figs. 4 and 5 that an increase of strain rate or ambient temperature gives fall-off transformed fraction at each level of the plastic strain. When the temperature rises to $573 \mathrm{~K}$, only a little martensitic fraction is transformed in impact process. 


\section{Conclusions}

This paper presented the quantitative prediction of the volume fraction of martensitic transformation in a austenitic steel that undergoes impact with high strain rate. The coupling relationship between strain, stress, strain rate, transformation rate and transformed fraction was derived from the OTC model and modified B-P equations, where the impact process was considered as an adiabatic and no entropy-increased process. The one-dimensional results were found to model and predict various experimental results obtained on 304 stainless steel under impact.

From the computational and experimental results the following conclusions are obtained:

(1) In the range of lower strain rate there is an obvious effect of strain rate on dynamic yield stress for 304-type steel, but in the range of higher strain rate this is not always the case. The effect of temperature on dynamic yield stress is obvious.

(2) An increase of strain rate or ambient temperature in martensitic transforming gives fall-off transformed fraction at each level of the plastic strain.

(3) The agreement between computational results and experimental results illustrates that the present formulations could account for the basic statistics and thermodynamic aspects of impact-induced martensites.

\section{Acknowledgements}

The authors acknowledge the financial support (No.19772042) from the National Science Foundation and (No.98010102) from the AEP Science Foundation of China.

\section{References}

[1] Stringfellow G, Parks DM, Olson GB. A constitute model for plasticity accompanying strain-induced martensitic transformation in metastable austenitic steel. Acta Metall Mater 1992;40:1703-16.

[2] Diani JM, Parks DM. Effect strain state on the kinetics of strain-induced martensite in steels. J Mech Phys Solids 1998;40(9):1613-35.

[3] Grujicic M, Sankaran N. Dispersed-phase martensitic transformation controlled deformation behavior of twophase metallic materials. Int J Solids Struct 1997;34:4421-46.

[4] Pack DC, Evans WM, James HJ. The propagation of shock waves in steel. Proc Phys Soc 1948;60:1.

[5] Olson GB, Tsuzaki K, Cohen M. Statistical aspects of martensitic nucleation. Mater Res Symp Proc 1987;57: $129-48$.

[6] Becker RZ. Phys 1926;547:7.

[7] Staudhammer KP, Frantz CE, Hecker SS, Murr LE. In: Meyers MA, Murr LE. Editors. Shock wave and highstrain-rate phenomena in metals, New York: plenum press, 1981. 